\title{
Remove the Ovarian Tumor, Cure the Encephalitis: Anti- NMDAR Encephalitis With Ovarian Teratoma in a Tertiary Hospital in Singapore
}

\author{
Ka Hee Chua a, c, Andy Wei Keat Tan ${ }^{a}$, Serene Liqing Limª, Wahab Syed Shahul Hameed ${ }^{\mathrm{b}}$, \\ Siew Ju See ${ }^{\mathrm{b}}$, Devendra Kanagalingam ${ }^{\mathrm{a}}$
}

\begin{abstract}
Anti-NMDAR encephalitis often causes profound neuropsychiatric symptoms in reproductive aged women. Diagnosis is based on identification of anti-NMDAR antibodies in the serum and cerebrospinal fluid (CSF), and such cases are commonly associated with ovarian teratomas. We present four cases of anti-NMDAR encephalitis with ovarian teratoma, and discuss their management, with special emphasis on the surgical approach. We propose laparotomy and systemic examination of both ovaries in such cases to avoid missing small teratomas.
\end{abstract}

Keywords: Anti-NMDAR encephalitis; Ovarian teratoma

\section{Introduction}

Anti-NMDAR encephalitis is a recently discovered disease entity. It has only been recognized since the last decade [1]. Many encephalitis cases that were previously thought of as idiopathic or psychiatric in nature may have been due to the anti-NMDAR antibody [2]. NMDA receptors are important in transmission of impulses at the synapse. Anti-NMDAR antibodies are IgG antibodies against the NR1 subunit of the NMDA receptor, and it results in a wide range of neuropsychiatric symptoms. It is often associated with an ovarian tumor, specifically teratomas. Treatment is by tumor removal and immunosuppression. Recovery is variable and can be prolonged, with episodes of relapse. We report four cases of anti-NMDAR encephalitis at a tertiary hospital in Singapore.

Manuscript accepted for publication January 11, 2017

aDepartment of Obstetrics and Gynaecology, Singapore General Hospital, Singapore

${ }^{b}$ Department of Neurology, Singapore General Hospital, Singapore

${ }^{\mathrm{c} C o r r e s p o n d i n g ~ A u t h o r: ~ K a ~ H e e ~ C h u a, ~ D e p a r t m e n t ~ o f ~ O b s t e t r i c s ~ a n d ~ G y n a e-~}$

cology, Singapore General Hospital, Singapore 169608, Singapore.

Email: Ka-hee.chua@mohh.com.sg

doi: https://doi.org/10.14740/jmc2734w

\section{Case Reports}

\section{Case 1}

A 22-year-old Indian woman presented with acute onset of bizarre behavior at work for 2 days. She was talking to herself, singing and dancing in her workplace. She was initially sent to a major psychiatric hospital for presumed psychosis, stabilized with intramuscular haloperidol and oral olanzapine, and transferred to a tertiary hospital to investigate for organic causes of her altered mental state. Two weeks prior to the onset of symptoms, she had a fever of $40{ }^{\circ} \mathrm{C}$ associated with headache, for which she saw the general practitioner and was given symptomatic treatment. She had no nausea, vomiting, photophobia or neck stiffness then, and recovered with symptomatic treatment only. On examination, her temperature was $36.7^{\circ} \mathrm{C}$, blood pressure was 129/77 $\mathrm{mm} \mathrm{Hg}$ and heart rate was 93 beats per minute. She refused to open her eyes, does not respond to calling, and was moving all four limbs spontaneously, albeit randomly. There was no other neurological deficit.

Her full blood count, electrolytes, liver panel, and thyroid function tests were unremarkable. An urgent brain CT did not detect any abnormality. In view of her recent history of fever, she was started on empirical antibiotics (intravenous ceftriaxone and vancomycin) and antiviral (intravenous acyclovir). Serological tests for cytomegalovirus, toxoplasmosis, herpes simplex, varicella zoster, measles, mumps, HIV, syphilis and hepatitis screen were negative, as were blood and urine cultures. Lumbar puncture yielded clear cerebrospinal fluid (CSF), and CSF microscopy, culture, cytology and infection screen were negative. Brain MRI under sedation was normal as well.

Her behavior deteriorated in the high dependency ward. She had episodes of loud and incoherent talking and laughing to herself, outbursts of shouting and physical violence at the nurses, and non-purposeful movement. She developed generalized tonic-clonic seizures, extension of her neck and all four limbs, and uprolling of her eyes, requiring intravenous diazepam. Electroencephalography (EEG) showed seizure activity in the left temporal lobe, and she was put on intravenous levetiracetam, phenytoin and oral topiramate.

A panel of antibodies was sent, including anti-NMDAR, anti-VGKC, anti-GAD and paraneoplastic antibodies $(\mathrm{Hu}$, Yo, Ri, CV2, amphihysin and PNMA2/TA). Anti-NMDAR 
returned as positive, with a titer of $1: 100$ in serum and 1:10 in CSF. Intravenous methylprednisolone was given for a total of 5 days. CT scan of the thorax, abdomen and pelvis was performed to look for a teratoma; it reported the ovaries to be normal and no other pathology. There was no improvement of her neuropsychiatric status after 5 days of methylprednisolone, despite decrease in serum anti-NMDAR titer to 1:32. Transabdominal and transrectal ultrasound was done (patient was virgo intacta) and showed a left ovarian cyst $51 \times 51 \times 44 \mathrm{~mm}$ with $16 \mathrm{~mm}$ echogenic area and few fine striations. Impression was a benign cystic teratoma. Ovarian tumor markers were normal.

She underwent a laparotomy ovarian cystectomy on the 37 th day from first onset of symptoms. Intraoperatively, the left ovary was enlarged with a normal appearance externally; a $2 \mathrm{~cm}$ dermoid cyst was apparent only upon incision and exploration of the ovary. Care was taken to avoid spillage of the cyst contents. Frozen section returned as mature teratoma. The right ovary was incised and explored and no cyst was found.

Postoperatively she was given routine analgesia and low molecular weight heparins for thromboprophylaxis. She had notable improvement within a few days: no seizures, no outbursts of aggressive behavior, transiently opens of eyes to calling. On the fifth day postoperation, she could respond to commands by squeezing fingers, wriggling of her toes, nodding and shaking her head. By 1 week postoperation, she could say her name and birthday, and could recognize her mother, and by 2 weeks, she could speak to her family and colleagues. However, she still had occasional abnormal behavior such as sitting on the floor during a physiotherapy session.

She was discharged home with her mother as caregiver. Final histology report showed a $1 \mathrm{~mm}$ focus of immature glial tissue and neuroepithelium, consistent with a grade 1 immature teratoma, stage 1. A multidisciplinary tumor board reviewed her case and recommended surveillance. By 6 weeks postoperation, she had no significant cognitive impairment and was back to her usual self. However, there was subtle difficulty in learning new information and she needed repeated exposures to learn. By 6 months postoperation, she had no more memory difficulties, and ultrasound pelvis showed normal ovaries.

\section{Case 2}

This is a 31-year-old Filipino female with no history of medical or psychiatric problems. She has a boyfriend of 4 years and a sister with her in Singapore.

She presented with 1-day history of abnormal behavior at work. She had incoherent speech, and was shouting loudly at patients and colleagues. She also had visual and auditory hallucinations as she talked of seeing computers and hearing strange sounds. On examination, she was agitated and restless. She had a temperature of $37^{\circ} \mathrm{C}$, blood pressure of $130 / 85 \mathrm{~mm}$ $\mathrm{Hg}$ and heart rate of 96 beats per minute. Her boyfriend who accompanied her reported altered behavior the night before. She was sending him incoherent text messages, at times total gibberish. He called her to ask if she had been drinking, but she seemed normal and relevant on the phone. However, subsequently she continued to send him messages that did not make sense.
She was given intramuscular midazolam and haloperidol to stabilize her in the emergency department and was admitted to the neurology ward. She developed seizures in the ward requiring benzodiazepine. There were frequent episodes of dyskinesia and purposeless movement, which resulted in her being restrained and sedated with phenytoin. Full septic workup, toxicology screen and infectious disease screen were negative. Full blood count, electrolytes, renal, liver and thyroid panels were normal. CT and MRI scans of the brain parenchyma and blood vessels were normal. Lumbar puncture was negative for infection and raised pressure. CSF histology showed $\mathrm{T}$ cell predominant activated lymphoid yield. EEG showed moderately severe diffuse encephalopathy.

Paraneoplastic antibody panel was sent, and anti-NMDAR was detected in both the serum and CSF, at titers of 1:32 and 1:100, respectively. CT scan of the thorax, abdomen and pelvis reported normal ovaries and no other pathology. Dedicated transvaginal ultrasound showed a $1 \mathrm{~cm}$ echogenic area in the right ovary, suspicious for a dermoid cyst. The uterus and left ovary were normal. Her case was discussed with the consultant gynecologist and it was decided for laparotomy exploration of bilateral ovaries. She was started on intravenous methylprednisolone and intravenous immunoglobulin (IVIG), and she underwent the surgery 8 days from onset of symptoms. Intraoperatively, both ovaries were bisected with cold knife, and examined systematically for cysts. A $1 \mathrm{~cm}$ cyst was found in her right ovary. Frozen section returned as mature cystic teratoma.

Postoperatively, there was no significant change in her neuropsychiatric status. She remained intubated in the intensive care unit. A tracheostomy was created in view of prolonged intubation. She continued to have seizure episodes, which needed maintenance midazolam, levetiracetam and phenytoin infusions. Two weeks after operation, she was started on weekly intravenous rituximab, but even then, she had minimal improvement. She had repeat lumbar punctures at 1 and 2 months post-surgery. Anti-NMDAR serum and CSF titer were $1: 320$ and $1: 32$ at 1 month, and $1: 100$ and $1: 32$ at 2 months. In view of refractory encephalitis and persistence of anti-NMDAR antibody, monthly cyclophosphamide injections were started. She slowly improved to a maximum GCS score of 7, where she can open eyes spontaneously and trace movement with her eyes, and respond to verbal questions by nodding. However, she had relapses and fluctuating GCS levels. Repeat transvaginal ultrasound did not reveal any more dermoid cysts. After 3 months, she was transferred back to a hospital in Philippines at the request of her family.

Final histology was that of a benign cystic teratoma with a single focus of neural tissue. There was difficulty in interpretation of the focus of neural tissue because it was very small; it may be ependymal tissue or immature neural tissue, and if it was the latter then it was a grade 1 tumor owing to its small size and single focus. Meticulous examination of the whole sample did not turn up any more neural tissue.

\section{Case 3}

A 26-year-old woman was brought to A\&E by her mother because of unresponsiveness to calling and one episode of in- 
voluntary jerking of upper limb. Her mother described her as being not herself for 2 days: constantly tearful, eating less and very forgetful. On examination, the patient was generally oriented, but she would get small details wrong. She could tell the time and date, but could not tell the year. She knew she was in hospital, but she gave the name of another hospital. Her temperature was $37.1^{\circ} \mathrm{C}$, blood pressure was $127 / 82 \mathrm{~mm} \mathrm{Hg}$, and pulse was 85 beats per minute. She had no obvious neurological deficit, but had blunted affect: slow to respond, poor eye contact, did not smile, had halting speech with frequent pauses and generally had no facial expressions. She was able to give a coherent history. For the past 2 days, she had been hearing voices at night but unable to discern what they were saying. There were no visual hallucinations but she pictured "bad" images in her head. She felt sad and emotional but she was not sure why she felt that way, and may even suddenly burst into tears. For the past week, she had difficulty in verbalizing and writing down her thoughts, and often spelled words incorrectly, but she was able to understand other people perfectly well. She also had memory impairment, and would forget things that were just heard a few minutes ago.

She had a history of twitching and fasciculations in her fingers and toes for 9 months for which she was managed conservatively. Electromyelography and nerve conduction studies were normal. She also had complaint of secondary amenorrhea for 6 months. Hormonal profile was normal, and ultrasound of pelvis showed a $1.9 \times 1.2 \times 1.1 \mathrm{~cm}$ echogenic avascular focus in the left ovary. Impression was that of either a dermoid cyst or a hemorrhagic cyst. She was given progestogen for withdrawal bleeding and advised to lose weight, as she had a BMI of 32. She had no previous mental or mood disorders, and no family history of psychiatric illness.

The patient had insight and was aware of her symptoms. She was anxious to be admitted and investigated. Blood investigations, septic screen, autoimmune screen and infectious disease screen were normal. CT and MRI scans of the brain were normal. A lumbar puncture was performed with an opening pressure of $10 \mathrm{~cm} \mathrm{H}_{2} \mathrm{O}$. CSF microscopy revealed more than $2 \mathrm{~g} / \mathrm{L}$ of protein; CSF culture, cytology and infection screen were normal. She was started on empirical intravenous acyclovir. An EEG showed intermittent slow, irregular activity over the left temporo-parieto-occipital region but no overt seizure activity. Her behavior was generally normal in the ward, even though she appeared quiet and withdrawn. A formal psychiatric referral was made.

Anti-NMDAR antibody returned as positive with a titer of 1:32 in the serum and 1:10 in the CSF. IV methylprednisolone was started and continued for 5 days. Transabdominal and transrectal ultrasound of pelvis showed an enlarged left ovary with an echogenic mass and central cystic area of $3.8 \times$ $2.5 \times 2.5 \mathrm{~cm}$, likely a dermoid cyst. The uterus and right ovary were normal, and ovarian tumor markers were not raised. She underwent a laparoscopic ovarian cystectomy on day 7 from onset of symptoms. Intraoperatively a $4 \mathrm{~cm}$ dermoid cyst was found in the left ovary and endometriotic spots were present in the pouch of Douglas. The rest of the pelvis was normal. There was inadvertent spillage of the cyst contents during the cystectomy and the specimen was removed in a bag.

She was fed on the day of operation and made an unevent- ful recovery. There was gradual but noticeable improvement in her memory and speech, and she was discharged on the fourth day postoperation. Final histology showed mature cystic teratoma. Repeat ultrasound done 3 months postoperation showed a non-specific $1.3 \times 0.9 \times 1.1 \mathrm{~cm}$ hyperechoic area in the left ovary with no obvious vascularity. By this time, she had regained normal speech, memory and behavior.

\section{Case 4}

A 31-year-old lady presented to another hospital with headache and photophobia. She was diagnosed with meningitis and started on intravenous ceftriaxone, metronidazole and acyclovir. CT scan of the brain showed mildly increased leptomeningeal enhancement in both cerebral hemispheres but no focal rim-enhancing lesion. However, she declined lumbar puncture and discharged against advice after 3 days. She came to our hospital 2 days later with worsening symptoms of headache, photophobia, neck pain, nausea and vomiting. Her temperature was $37.5^{\circ} \mathrm{C}$, blood pressure was $128 / 74 \mathrm{~mm} \mathrm{Hg}$ and pulse was 97 beats per minute. On examination, she was lucid but Kernig's sign was positive. An urgent CT of brain showed similar finding of mild, equivocal leptomeningeal enhancement in the frontal basal regions, with no overt parenchymal lesion. Presumptive diagnosis of partially treated bacterial meningitis was made, with a differential being tuberculous meningitis, and she was restarted on intravenous antibiotics.

Despite IV antibiotics and anti-tuberculous treatment, her condition deteriorated. The next day, she developed bilateral sixth cranial nerve 6 palsies, diplopia and visual hallucinations. The following day, she became restless, uncooperative, and exhibited confused speech. Over the next few days, she became more and more drowsy and unresponsive to commands. MRI of brain showed mild sulcal FLAIR hyperintensity predominantly in bilateral parietal occipital lobes, associated with mild diffuse leptomeningeal enhancement, in keeping with submitted history of meningitis. One week after admission, she was prophylactically intubated and transferred to intensive care unit as she became unconscious with a Glasgow coma score of 3 .

CSF microscopy showed leukocytosis. Bacterial cultures, Mycobacterium tuberculosis DNA, viral tetraplex, neurotrophic virus, herpes simplex virus DNA, Enterococcus RNA, Cryptococcus antigen, cytology, VDRL and fungal cultures were negative in the CSF. EEG was normal. She developed dyskinesia, evidenced by chewing on the endotracheal tube, and dysautonomia, with pulse fluctuating from 45 to 145 beats per minute, long periods of bradycardia at 50 beats per minute, and hyperthermia of $40{ }^{\circ} \mathrm{C}$. She underwent a tracheostomy 1 week after transfer to ICU in anticipation of prolonged intubation.

CT scan of the thorax, abdomen and pelvis was performed to rule out paraneoplastic causes, as well as paraneoplastic antibody panel. CT was unremarkable for the pelvic organs, except for a $2 \mathrm{~cm}$ corpus luteal cyst in the left ovary. Anti-NMDAR antibodies returned as positive. A transvaginal ultrasound showed normal uterus, a $2 \mathrm{~cm}$ left ovarian corpus luteum and an $8.1 \times$ $4.3 \times 4.7 \mathrm{~cm}$ bilobulated mass with multiple echogenic foci in the right adnexa. Impression was that of a right dermoid cyst.

She was put on intravenous dexamethasone for 2 weeks 
Table 1. Comparison of Four Cases

\begin{tabular}{|c|c|c|c|c|c|}
\hline & Case 1 & Case 2 & Case 3 & Case 4 & Summary \\
\hline Age (years) & 22 & 31 & 26 & 31 & $\begin{array}{l}27.5 \\
\text { (mean) }\end{array}$ \\
\hline Seizures & $\mathrm{Y}$ & Y & $\mathrm{N}$ & $\mathrm{N}$ & $2 / 4$ \\
\hline Dyskinesia & $\mathrm{Y}$ & $\mathrm{Y}$ & $\mathrm{N}$ & $\mathrm{Y}$ & $3 / 4$ \\
\hline MRI brain abnormality & $\mathrm{N}$ & $\mathrm{N}$ & $\mathrm{N}$ & $\mathrm{Y}$ & $1 / 4$ \\
\hline Ovarian pathology seen on CT & $\mathrm{N}$ & $\mathrm{N}$ & Not done & $\mathrm{N}$ & $3 / 3$ \\
\hline Size of tumor seen on US $(\mathrm{cm})$ & 5.1 & 1 & 3.8 & 8.1 & \\
\hline $\begin{array}{l}\text { Time from symptom onset to surgery } \\
\text { (days) }\end{array}$ & 37 & 8 & 7 & 28 & 20 (mean) \\
\hline Type of surgery & $\begin{array}{l}\text { Laparotomy } \\
\text { ovarian cystectomy }\end{array}$ & $\begin{array}{l}\text { Laparotomy } \\
\text { ovarian cystectomy }\end{array}$ & $\begin{array}{l}\text { Laparoscopic ovarian } \\
\text { cystectomy }\end{array}$ & $\begin{array}{l}\text { Laparotomy unilateral } \\
\text { salpingo-oophorectomy }\end{array}$ & \\
\hline Size of tumor, intraoperative $(\mathrm{cm})$ & 2 & 1 & 4 & 5 & \\
\hline Frozen section result & Benign & Benign & Not performed & Benign & \\
\hline Final histology & Malignant & Malignant & Benign & Malignant & \\
\hline Intravenous steroid & $\mathrm{Y}$ & $\mathrm{Y}$ & $\mathrm{Y}$ & $\mathrm{Y}$ & $4 / 4$ \\
\hline IVIG & $\mathrm{N}$ & Y & $\mathrm{N}$ & Y & $2 / 4$ \\
\hline Second line immunosuppressants & $\mathrm{N}$ & Y & $\mathrm{N}$ & $\mathrm{Y}$ & $2 / 4$ \\
\hline
\end{tabular}

and IVIG for 1 week, but there was no improvement in her condition. It was then decided for excision of the ovarian tumor. An uncomplicated laparotomy and salpingo-oophorectomy of the right adnexa was performed, 4 weeks after onset of symptoms. Intraoperatively, a $5 \mathrm{~cm}$ cyst with solid areas was found in the right ovary. Frozen section returned as benign cyst. There was no ascites, and the uterus and contralateral ovary were normal.

She had a stormy postoperative recovery, complicated by sepsis secondary to pneumonia and urinary candidiasis. There was no improvement in her conscious status initially; she remained unresponsive and was started on second line immunosuppressive agent (rituximab) 2 weeks after the operation. Three weeks after the operation, she started having gradual improvement. First the dyskinesia stopped, then she started responding to verbal stimuli, and subsequently she attempted to sit up in bed. She stayed for a further month in high dependency, and was monitored for another month in the general ward before being transferred to rehabilitation medicine facilities. Oral azathioprine was started and planned to be continued for 2 years as maintenance immunosuppressive therapy.

Final histology returned as grade 2 immature teratoma with intact capsule, containing immature neuroepithelium and neuroglial tissue. She was started on chemotherapy 3 months postoperation, after satisfactory neurological recovery. She completed six cycles of etoposide and cisplatin. At 4 months postoperation, she had recovered fully and at 5 years postoperation, she has had no relapse of her symptoms.

\section{Discussion}

Limbic encephalitis is a form of inflammatory encephalitis that affects the limbic system. Many antibodies have been found to cause this condition, and have been commonly associated with tumors of the lung, testis, ovaries, breast and non-Hodgkin's lymphoma, hence the term paraneoplastic encephalitis. AntiNMDAR has been found to be associated with ovarian teratoma. Onset of symptoms is typically abrupt, and they include memory deficit, seizures, dyskinesias and language dysfunction. Severe psychiatric symptoms are common and prominent, including behavioral change, anxiety, hallucinations and schizophrenia. Thus, majority of them are seen at first instance by a psychiatrist, and a smaller proportion are seen initially by the neurologist [1]. Only when neurological symptoms surface such as seizures or dyskinesias are they then referred to the 
neurologist.

It was noted previously that these patients commonly have a prodromal viral-like illness a few weeks prior to the acute deterioration. A search for infective agents in the blood and CSF in our patients turn out negative. These symptoms could be an immune response to the endogenous antibodies from the ovarian teratoma. Another cause of anti-NMDAR antibodies is post-viral infections, most commonly herpes simplex [3]. In this case, autoantibodies are formed presumably from viral mediated destruction/inflammation of neuronal tissue. Empirical antivirals are often started while investigating the cause.

This condition tends to affect young people, even the pediatric population [4]. In a case series of 100 patients, the median age was 23 years [1]. The age range for our patient was from 22 to 31 years (Table 1). CSF studies, EEG and MRI may have abnormal but non-specific findings. CSF may show leukocytosis, increased protein content or oligoclonal bands. EEG may show slow, disorganized activity or frank seizure activity. MRI FLAIR sequence may show abnormalities in certain brain regions. Definite diagnosis depends on isolation of antiNMDAR antibody in either serum or CSF. As our case series demonstrates, laboratory and radiological findings may vary in affected individuals.

Antibody titers appear to correlate with severity of symptoms and response to treatment. One study measured serum and CSF titers at pre-treatment phase, immediately post-treatment (treatment consists of unilateral salpingo-oophorectomy, methylprednisolone and plasma exchanges), and 3 months post-treatment. They demonstrated that antibody titers decreased with time post-treatment, and CSF titers seem to correlate better with the symptomatology compared to serum titers [5]. In our series, the patient with the mildest symptoms and most rapid recovery also had the lowest antibody titers (case 3 ). We do not repeat titers routinely post-treatment, except in cases of refractory encephalitis.

The tumors responsible for the symptoms may be very small in size. In our series, two of the teratomas were 1 and 2 $\mathrm{cm}$ each. They may be easily missed on imaging. CT scan is often the first line imaging modality to look for ovarian teratomas. However, if a CT scan is negative, we propose that a transvaginal or transrectal ultrasound scan be done. In the three cases where $\mathrm{CT}$ of thorax, abdomen and pelvis were performed to look for tumors causing paraneoplastic syndrome, ovarian teratomas were not seen on CT scan, but were subsequently diagnosed on ultrasound. Transrectal or transvaginal ultrasound scans may be superior to CT scans in detecting small ovarian tumors as the proximity of the ultrasound probe to the lesion allows careful and thorough examination of the ovaries. Ovarian teratomas also have a distinct appearance on ultrasound scan allowing them to be easily identified.

The small size of the tumors may make intra-operative recognition difficult. In the two cases with tumors of 1 and $2 \mathrm{~cm}$, the external appearance of the ovary was normal, and the tumor was only found on incision and exploration of the ovary. One study reported bilateral salpingo-oophorectomy of radiologically and macroscopically normal ovaries in a patient refractory to treatment, and found a $0.7 \mathrm{~cm}$ mature teratoma on histopathology; the patient subsequently recovered [6]. Ovarian teratomas causing anti-NMDAR encephalitis can occasion- ally be bilateral [7], so we suggest incising the contralateral ovary, even if it appears outwardly normal. Proper exploration of the ovary includes using palpation to detect small tumors. This will not be possible in laparoscopy. We use cold knife to bisect the ovary instead of diathermy devices. This minimizes thermal damage to the ovary and depletion of the follicular pool. Many of these women are in the childbearing age and future fertility is an important consideration for them. We use absorbable sutures for hemostasis and to reconstruct the ovary, and avoid diathermy coagulation for hemostasis.

In a systematic review of 174 cases of anti-NMDAR encephalitis with ovarian teratomas, $26 \%$ were immature [8]. Generally, immature teratomas comprise less than $1 \%$ of all teratomas. They are malignant tumors and require proper surgical staging. Inadvertent spillage of cyst contents and dissemination of a potentially malignant tumor is also a concern. In view of the high incidence of immature tumors in this condition and the severe consequences of not removing even a small focus of tumor, we propose that laparotomy be the mainstay of treatment rather than laparoscopy. In our series, three out of four patients had immature teratoma. An alternative approach would be to do a unilateral salpingo-oophorectomy. This would avoid spillage of cyst contents and can be performed laparoscopically. However, most of these patients are young and conservation of ovarian tissue would be an important consideration. The risks and benefits of cystectomy versus salpingo-oophorectomy should be discussed in detail with the patient.

Our experience with frozen section in these cases has been unsatisfactory. The advantage of frozen section is twofold: it allows us to offer ovarian conserving surgery, and if frozen section returns as malignant, it allows us to do a staging procedure at the same instance without needing to undergo a second operation. However, in all our cases, the final histology was discordant with the initial frozen section. This could be due to the small size of the immature component; in our first two cases, the foci of immature neurotissue were $1 \mathrm{~mm}$ or less. One of our patients had to undergo adjunctive chemotherapy. More evidence is needed before frozen section can be recommended or discouraged in these cases.

Treatment of this condition includes excision of the ovarian teratoma and immunosuppressive therapy. Tumor removal in combination with immunosuppressive therapy results in better and more rapid recovery compared to immunosuppression alone [1]. In two of our cases, pre-operative intravenous methylprednisolone over at least 5 days did not elicit any improvement. The patients only started recovery after excision of the tumor. Recovery may be prolonged and recurrences have been documented, hence long-term follow-up is necessary. It is notable that three of our four patients made a full recovery. The remaining patient showed clinical improvement but had residual neurological deficits at the time when she was transferred to another institution.

\section{Conclusion}

Anti-NMDAR encephalitis is a rare cause of profound neuropsychiatric symptoms. Diagnosis is by isolation of anti- 
NMDAR antibodies in the serum or CSF and ultrasound is the preferred imaging modality to look for ovarian teratomas. We suggest laparotomy, ovarian cystectomy and proper exploration of bilateral ovaries in young patients. In older patients where fertility is not an issue, unilateral or bilateral salpingooophorectomy should be considered. We believe laparotomy to be the better approach in most of these women compared to laparoscopy, as a significant proportion of these tumors have immature elements and are, hence, malignant. Laparotomy also allows careful palpation of the ovaries as complete removal of even small teratomas is the key to eventual recovery of these patients. Awareness of this entity is essential to ensure that a search for ovarian teratomas is performed and timely surgery, an important aspect of the treatment, is carried out.

\section{References}

1. Dalmau J, Tuzun E, Wu HY, Masjuan J, Rossi JE, Voloschin A, Baehring JM, et al. Paraneoplastic anti-Nmethyl-D-aspartate receptor encephalitis associated with ovarian teratoma. Ann Neurol. 2007;61(1):25-36.

2. Pruss H, Dalmau J, Harms L, Holtje M, Ahnert-Hilger G, Borowski K, Stoecker W, et al. Retrospective analysis of NMDA receptor antibodies in encephalitis of unknown origin. Neurology. 2010;75(19):1735-1739.

3. Pruss H, Finke C, Holtje M, Hofmann J, Klingbeil C,
Probst C, Borowski K, et al. N-methyl-D-aspartate receptor antibodies in herpes simplex encephalitis. Ann Neurol. 2012;72(6):902-911.

4. Hacohen Y, Wright S, Waters P, Agrawal S, Carr L, Cross H, De Sousa C, et al. Paediatric autoimmune encephalopathies: clinical features, laboratory investigations and outcomes in patients with or without antibodies to known central nervous system autoantigens. J Neurol Neurosurg Psychiatry. 2013;84(7):748-755.

5. Seki M, Suzuki S, Iizuka T, Shimizu T, Nihei Y, Suzuki N, Dalmau J. Neurological response to early removal of ovarian teratoma in anti-NMDAR encephalitis. J Neurol Neurosurg Psychiatry. 2008;79(3):324-326.

6. Dabner M, McCluggage WG, Bundell C, Carr A, Leung Y, Sharma R, Stewart CJ. Ovarian teratoma associated with anti-N-methyl D-aspartate receptor encephalitis: a report of 5 cases documenting prominent intratumoral lymphoid infiltrates. Int J Gynecol Pathol. 2012;31(5):429-437.

7. Tantipalakorn C, Soontornpun A, Pongsuvareeyakul T, Tongsong $\mathrm{T}$. Rapid recovery from catastrophic paraneoplastic anti-NMDAR encephalitis secondary to an ovarian teratoma following ovarian cystectomy. BMJ Case Rep. 2016;2016.

8. Acien P, Acien M, Ruiz-Macia E, Martin-Estefania C. Ovarian teratoma-associated anti-NMDAR encephalitis: a systematic review of reported cases. Orphanet J Rare Dis. 2014;9:157. 